\title{
Can postpyelonephritic renal scarring be prevented?
}

\author{
Tryggve Nevéus
}

Received: 26 September 2012 / Accepted: 27 September 2012 / Published online: 20 October 2012

(C) IPNA 2012

\begin{abstract}
Pyelonephritis in childhood may, in the worst cases, lead to long-term cardiovascular morbidity due to tubulointerstitial renal scarring. Renal damage is the end result of an interplay between (1) urinary tract anatomy and function, (2) bacterial virulence factors, and (3) the host innate immune system, which on the one hand manages bacterial clearance, but on the other causes tubulointerstitial inflammation, which underlies the renal scarring. It is unclear how common postpyelonephritic scarring is, and how many of the "scars" in fact represent congenital renal hypoplasia. We do, however, know that some situations have an increased risk for scars, i.e., large renal-uptake defects on initial renal scintigraphy or pyelonephritis in young girls with dilating vesicoureteral reflux. It seems logical that antiinflammatory or antioxidant therapy given concomitantly with antibiotics should lower the risk of postpyelonephritic scarring. Animal studies give some support to this idea, but research on humans has been surprisingly scant. In this issue of Pediatric Nephrology, we publish a study that indicates that antioxidant therapy with vitamin $\mathrm{A}$ or $\mathrm{E}$ given to children with pyelonephritis may indeed lower the risk for renal scarring. This is a track that needs to be pursued further.
\end{abstract}

Keywords Pyelonephritis $\cdot$ Inflammation ·

Postpyelonephritic scarring $\cdot$ Antioxidants $\cdot$ Corticosteroids

\section{Introduction}

The relationship between uropathogenic bacteria and the human being is peculiar in several respects. The adaptive immune defense (lymphocytes, antibodies, etc.) is not important in the protection from urinary tract infections (UTIs). Pyelonephritis is not a major problem in AIDS

T. Nevéus $(\bowtie)$

Department of Women's and Children's Health,

Uppsala University,

Uppsala, Sweden

e-mail: tryggve.neveus@kbh.uu.se patients or patients receiving heavy immunosuppression. In fact, probably the most important protection against bacteria is the ability to expel them into the exterior before they get the chance to proliferate too much, i.e., to have normal lower urinary (LUT) tract function and anatomy. Furthermore, the damage that may or may not result from attacks by these bacteria is caused not by the bacteria but by the local inflammatory response. Consequently, there should be much research into ways to reduce the local inflammatory response in order to reduce the risk of renal scarring, but there is not. This is perplexing.

\section{Mechanisms of renal scarring after pyelonephritis}

The mechanisms leading to renal inflammation and tubulointerstitial fibrosis that may be the consequence of an upper UTI are incompletely known, but knowledge is increasing. The emerging picture is one of interaction between bacterial virulence, host LUT anatomy/function, and host innate immune response. The adaptive immune response plays no or only a very limited role.

The first step is, of course, bacterial access to the renal pelvis. This is facilitated by vesico-uretheral reflux (VUR) among infants and small children [1] or residual urine in older children [2]. The next step is bacterial adhesion to the urothelium. This is facilitated via the virulence factors of the bacteria, and foremost among them the $\mathrm{P}$ fimbriae of uropathogenic Escherichia coli [3]. This elicits intracellular signaling via Toll-like receptor 4 (TLR4) on the urothelial cell membrane, which, in turn, causes the cell to produce and release inflammatory mediators such as complement factors, cytokines, and adhesion molecules [4]. The neutrophil leukocytes that are attracted to the tubulointerstitium by these mediators lead to the release of toxic enzymes and an increase of oxygen-free radicals.

The result is both bacterial clearance and tubulointerstitial inflammation [5]. The latter may later be suppressed or result in fibrosis. We do not know why some children get 
scars and some do not [6], but one factor is the activity of the IL-8 receptor CXCR, which mediates the movement of the neutrophils from the interstitium into the urine [7].

\section{Epidemiology and consequences}

The classic worst-case scenario is the child whose kidneys are scarred after pyelonephritis early in life and who then receives more infections, more scarring, hypertension, proteinuria, and finally progressive renal failure and the need for renal replacement therapy.

This situation is now, luckily, rare in many parts of the world. Instead, the picture has become very confused. We do not know:

(1) how common post-pyelonephritic renal scarring is

(2) how many of the so-called scars are in fact instances of congenital renal hypoplasia

(3) which scars/hypoplasias represent a long-term risk for the patient.

Figures for the risk of developing renal damage after pyelonephritis vary wildly. Estimations from as low as $8 \%$ [8] up to two-thirds have been made [9]. A recent meta-analysis, however, puts the risk in the lower range [10]. There are several reasons for the varying estimates. Dimercaptosuccinic acid (DMSA) scans are assessed differently by different investigators, and there is a lack of firm guidelines as to which scan is to be regarded as pathological and which is not. However, there are also secular trends and global differences. The risk for renal scarring was probably higher a few decades ago than it is now [10] and the situation can be suspected to differ between low- and high-income populations.

What we do know, however, is that some situations are associated with a higher-than-normal risk acquired renal scarring. Pyelonephritis with large uptake defects on acute DMSA renal scintigraphy is one such situation. Pyelonephritis in girls below age 2 with dilated VUR is another [11].

It has become increasingly clear that all of the renal scars that are found after a pyelonephritis are not damage caused by the infection [12]; perhaps not even the majority. Congenital renal hypoplasia is common among kidneys attached to refluxing ureters. The emerging picture is that renal hypoplasia is probably common, especially in boys with dilating reflux, whereas acquired scarring is typical for girls with recurrent febrile UTIs [13]. In the individual case, however, it is often impossible to differ between the two conditions.

Most of us would guess that the risk for long-term morbidity such as hypertension, proteinuria, complications of pregnancy, and deteriorating renal function would be higher in cases of acquired renal scarring than in congenital renal hypoplasia, but this is not proven [12]. We do not really know how big the renal scar needs to be for us to be worried about the child's future. Many children - at least in industrialized countries - are therefore undergoing unnecessary regular check-ups year after year, whereas other children with potentially harmful renal damage are missed.

While much effort has been put into looking for factors predisposing a person to relapsing febrile UTIs, such as VUR and bladder or bowel dysfunction, and progress has been made regarding how to prevent UTIs in children at increased risk, there are still only a handful of researchers who have seriously addressed the question about what can be done to prevent the development of renal scars once a pyelonephritis has occurred.

\section{Anti-inflammatory or antioxidant therapy}

The assumption that anti-inflammatory or antioxidant therapy may reduce the risk for renal scarring is not far-fetched, given the mechanisms behind pyelonephritic renal damage described above. If effective and safe treatment against the harmful effects of the neutrophil attack against the renal interstitium could be given concomitantly with the antibiotic treatment against the bacteria, much would be won, at least in children at increased risk. Such risk groups could be infants, young girls, or patients with an unfavorable urothelial receptor profile (high TLR4, low CXCR).

One track to follow that seems appealing is to lower the urothelial cytokine release and neutrophil recruitment with corticosteroid therapy. The rationale for this strategy is that the crucial second step in the local inflammatory reaction, i.e., the urothelial cellular response to the adhesion of bacteria to TLR4, is downgraded by corticosteroids [14].

This concept has been supported, first by animal studies $[15,16]$, and lately in an elegant placebo-controlled study on children with a first febrile UTI [17]. In this study, Huang et al. showed that the risk for renal scarring after 6 months in children who had a severe pyelonephritis (the severity defined as large renal uptake defects on initial DMSA scintigraphy or renal swelling visible on ultrasound) was approximately halved if oral methylprednisolone was given for 3 days concomitantly with antibiotic therapy.

Another possible option is to use antioxidants such as vitamin A or E. This line of research also has a logical appeal - given the role of free oxygen radicals in the inflammatory damage to the tubulointerstitium - but has been almost completely ignored by researchers in the Western world.

There is data to support this strategy as well. Both vitamin $\mathrm{A}$ and $\mathrm{E}$ seem to be able to limit pyelonephritic renal damage in animals [18-20], and two recent open, randomized studies from Iran both indicate that vitamin A may reduce scarring in humans as well [21, 22].

The antioxidant strategy is highlighted again by the study by Sobouti et al. in the present issue of Pediatric Nephrology 
[23]. In this work, children with pyelonephritis between 1 month and 10 years of age were given either antibiotics only, antibiotics + vitamin A, or antibiotics + vitamin E. Both vitamin treatments were found to be protective against the development of renal scarring.

The study deserves attention but should not be taken at face value, since there are limitations. The delineation of the patient group was somewhat vague, treatment was not blinded, and the incidence of renal damage in the controls (77\%) was suspiciously high (furthermore, the antibiotic strategy, with long-term indiscriminate broad-spectrum antibiotic prophylaxis, would be applauded by only very few experts, but this is not the issue here). Still, if this study could be replicated as a proper multi-center, placebocontrolled study, we might certainly be onto something.

\section{Future perspectives}

Even when the uncertainties regarding the true frequency and long-term consequences of acquired pyelonephritic scarring mentioned above are taken into account, it is clear that much would be won if a harmless way to diminish the risk of such renal damage were found. If it could be confirmed that anti-inflammatory or antioxidant therapy, given in conjunction with antibiotic treatment, really diminishes the risk for long-term renal sequelae, then we may have found a way to prevent future cardiovascular and renal morbidity for a quite large patient group.

As mentioned above, I find it surprising that more work has not been done in this field. The reason for this inactivity may be prejudice against research from non-Western countries and/or low incentives from the medical industry. It is usually difficult to find industry sponsorship for clinical trials involving old, cheap drugs that are to be given for only a limited time. Anyway, the Turkish and Iranian researchers looking into vitamin therapy deserve our respect, and the study on steroid treatment by Huang et al. needs to be replicated in a larger patient population and then perhaps implicated in clinical practice [17].

Personally, I feel more attracted by the steroid strategy than the antioxidant strategy. This treatment affects the potentially harmful inflammatory response at an earlier stage than the antioxidants and we can be confident that oral steroids given for, say, 3 days is harmless. However, one may also argue that the antioxidants, with their influence on apoptosis, are closer to the core of the problem.

So, if I may venture a guess about how we will manage pyelonephritis in childhood in the future, the treatment protocols may include a strategy such as the following:

(A) Start antibiotic therapy as soon as pyelonephritis is considered likely.
(B) After 2-3 days, if (1) clinical situation is stable, (2) bacterial culture confirms infection with bacteria sensitive to the antibiotics given, and (3) the patient belongs to a risk group for renal damage, then

(C) add oral corticosteroids for 3 days

\section{References}

1. Koyle MA, Elder JS, Skoog SJ, Mattoo TK, Pohl HG, Reddy PP, Abidari JM, Snodgrass WT (2011) Febrile urinary tract infection, vesicoureteral reflux, and renal scarring: current controversies in approach to evaluation. Ped Surg Int 27(4):337-346

2. Rudaitis S, Pindziene B, Jievaltas M, Uktveris R, Kevelaitis E (2009) Recurrent urinary tract infection in girls: do urodynamic, behavioral and functional abnormalities play a role? J Nephrol 22 (6):766-773

3. Johnson JR, Roberts PL, Stamm WE (1987) P fimbriae and other virulence factors in Escherichia coli urosepsis: association with patients' characteristics. J Inf Dis 156:225-229

4. Ragnarsdóttir B, Svanborg C (2012) Susceptibility to acute pyelonephritis or asymptomatic bacteriuria: host-pathogen interaction in urinary tract infections. Pediatr Nephrol. doi:10.1007/s00467-0112089-1

5. Mak RH, Kuo HJ (2006) Pathogenesis of urinary tract infection: an update. Curr Opin Ped 18(2):148-152

6. Hewitson JD (2009) Renal tubulointerstitial fibrosis: common but never simple. Am J Physiol Renal Physiol 296(6):F1239-1244

7. Godaly G, Hang L, Frendeus B, Svanborg C (2000) Transepithelial neutrophil migration is CXCR1-dependent in vitro and is defective in IL-8 receptor mutant mice. J Immun 165:5287-5294

8. Hoberman A, Wald ER, Hickey RW, Baskin M, Charron M, Majd M, Kearney DH, Reynolds EA, Ruley J, Janosky JE (1999) Oral versus initial intravenous therapy for urinary tract infections in young febrile children. Pediatrics 1104:79-86

9. Orellana P, Baquedano P, Rangarajan V, Zhao JH, Eng NDC, Fettich J, Chaiwatanarat T, Sonmezoglu K, Kumar D, Park YH, Samuel AM, Sixt R, Bhatnagar V, Padhy AK (2004) Relationship between acute pyelonephritis, renal scarring, and vesicoureteral reflux. Results of a coordinated research project. Pediatr Nephrol 19:1122-1126

10. Shaikh N, Ewing AL, Bhatnagar S, Hoberman A (2010) Risk of renal scarring in children with a first urinary tract infection: a systematic review. Pediatrics 126:1084-1091

11. Brandström P, Jodal U, Nevéus T, Sixt R, Stokland E, Hansson S (2010) The Swedish reflux trial in children. IV. Renal damage. J Ped Urol 10(6(Suppl 1)):33-4

12. Mattoo TK (2011) Vesicoureteral reflux and reflux nephropathy. Adv Chron Kidney Dis 18(5):348-354

13. Wennerström M, Hansson S, Jodal U, Stokland E (2000) Primary and acquired renal scarring in boys and girls with urinary tract infection. J Pediatr 136:30-34

14. Moynagh PN (2003) Toll-like receptor signalling pathways as key targets for mediating the anti-inflammatory and immunosuppressive effects of glucocorticoids. J Endocrinol 179(2):139-144

15. Haraoka M, Matsumoto T, Takahashi K, Kubo S, Tanaka M, Kumazawa J (1994) Suppression of renal scarring by prednisolone combined with ciprofloxacin in ascending pyelonephritis in rats. J Urol 151:1078-1080

16. Pohl HG, Rushton HG, Park JS, Chandra R, Majd M (1999) Adjunctive oral corticosteroids reduce renal scarring: the piglet 
model of reflux and acute experimental pyelonephritis. J Urol 162 (3 Pt 1):815-820

17. Huang Y, Chen M, Chiu N, Chou H, Lin K, Chiou Y (2001) Adjunctive oral methylprednisolone in pediatric acute pyelonephritis alleviates renal scarring. Pediatrics 128:e496

18. Kavukçu S, Soylu A, Türkmen M, Sarioglu S, Büyükgebiz B, Güre A (1999) The role of vitamin A in preventing renal scarring secondary to pyelonephritis. BJU Int 83(9):1055-1059

19. Emamghorashi F, Owji SM, Motamedifar M (2011) Evaluation of effectiveness of vitamins $\mathrm{C}$ and $\mathrm{E}$ on prevention of renal scar due to pyelonephritis in rat. Adv Urol. doi:10.1155/2011/489496

20. Sadeghi Z, Kajbafzadeh AM, Tajik P, Monajemzadeh M, Payabvash S, Elmi A (2008) Vitamin E administration at the onset of fever prevents renal scarring in acute pyelonephritis. Pediatr Nephrol 23(9):1503-1510

21. Dalirani R, Yousefi Zoshk M, Sharifian M, Mohkam M, Karimi A, Fahimzad A, Varzandefar M (2011) Role of vitamin A in preventing renal scarring after acute pyelonephritis. Iran J Kidney Dis 5 (5):320-323

22. Ayazi P, Moshiri SA, Mahyar A, Moradi M (2011) The effect of vitamin A on renal damage following acute pyelonephritis in children. Eur J Pediatr 170(3):347-350

23. Sobouti B, Hooman N, Movahed M (2012) The effect of vitamin E or vitamin A on the prevention of renal scarring in children with acute pyelonephritis. Pediatr Nephrol. doi:10.1007/s00467-0122308-4 